\title{
Witnessed apnoeas in a 6-yr-old boy: a brain teaser
}

\section{Case history}

A 6-yrold boy was admitted to our paediatric sleep lab because of witnessed apnoeas by his parents. There were no difficulties falling asleep, nocturnal awakenings nor excessive daytime sleepiness or tiredness.

He was a boy of nonconsanguine parents with normal developmental milestones. His past medical history included snoring which disappeared after adenotonsillectomy at the age of 4 yrs, asthma and allergic rhinitis for which he was on salmeterol and fluticason, cetirizine and mometason. He was also seen 6 months prior to admission because of headache after physical effort without vomiting. Blood pressure, EEG, dilated fundus examination and renal workup were all normal. A brain MRI was also performed in another hospital of which we had a written report stating that it was normal. The complaints of headache spontaneously resolved over the next months.

Familial history was negative for sleep, respiratory, neurological and other relevant medical disorders.

Clinical and neurological examination was normal. Length was on the 25th percentile and weight on the 50th percentile. His body mass index was $16.5 \mathrm{~kg} \cdot \mathrm{m}^{-2}$.

A singlenight, full polysomnography was performed to rule out residual obstructive sleep apnea syndrome after adenotonsillectomy (fig. 1).

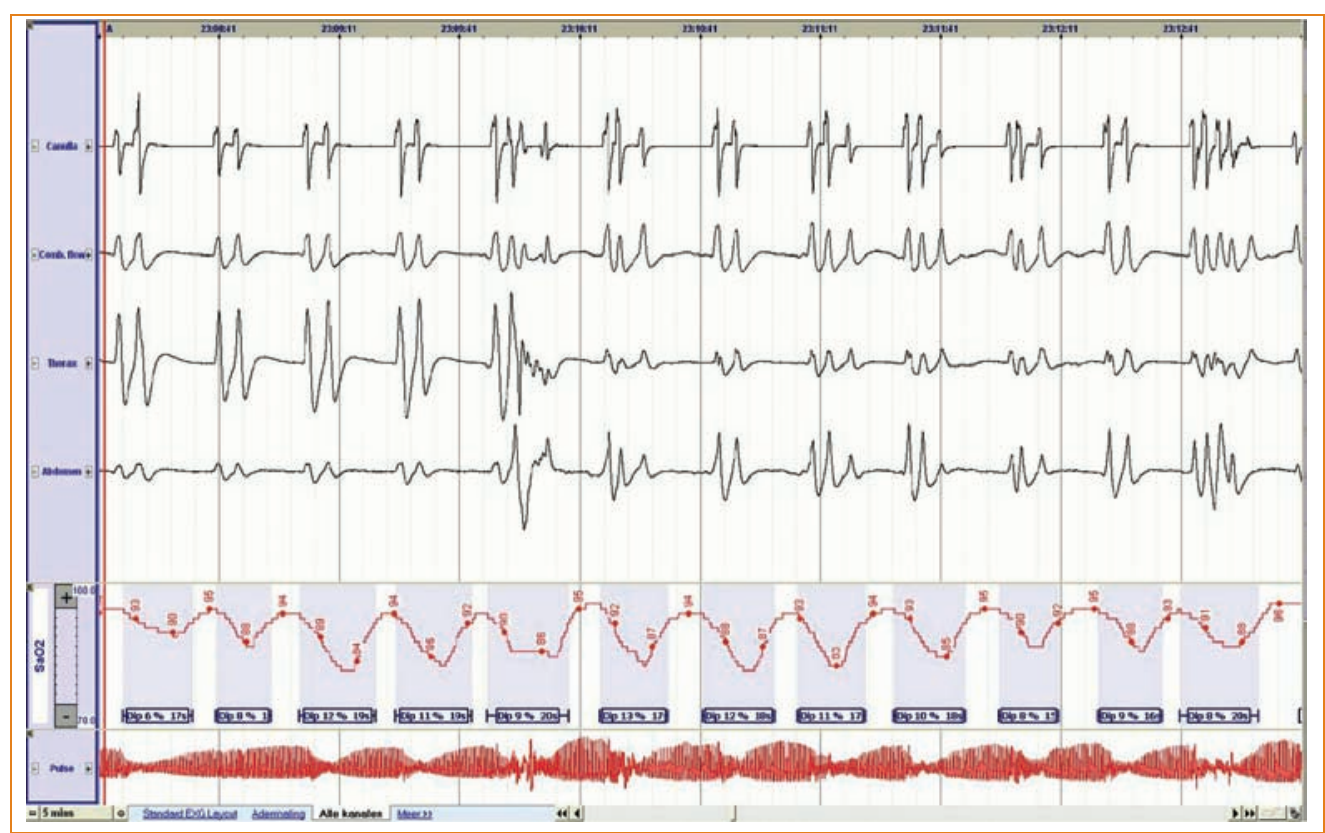

Figure 1

Polysomnography results. 


\section{Answer 1: \\ The tracing shows the presence of periodic breathing. Periodic breathing is present if there are $>$ three episodes of central apnoea lasting $>3$ seconds separated by no more than 20 seconds of normal breathing.}

We performed a polysomnography and it was repeated because to confirm our findings. The results are summarised in table 1.

\section{Table 1 Result of polysomnography (PSG)}

\begin{tabular}{|c|c|c|}
\hline & PSG1 & PSG2 \\
\hline Sleep onset latency & 4 mins & 29.6 mins \\
\hline Sleep-onset rapid eye movement & 289.0 mins & 220.0 mins \\
\hline Sleep histogram & Sleep fragmentation & Sleep fragmentation \\
\hline Central apnoea index & 40.1 per $h$ & 37.9 per h \\
\hline Apnoea-hypopnoea index & 0.7 per $\mathrm{h}$ & 1.1 per h \\
\hline Periodic breathing & $\begin{array}{l}25 \% \text { of total sleep } \\
\text { time }\end{array}$ & $\begin{array}{l}39.8 \% \text { of total sleep } \\
\text { time }\end{array}$ \\
\hline $\mathrm{SaO}_{2}$ & $95 \%$ & $95.0 \%$ \\
\hline $\mathrm{SaO}_{2}$ nadir & $82.0 \%$ & $79.0 \%$ \\
\hline Total sleep time at $\mathrm{SaO}_{2}<95 \%$ & $7.3 \%$ & $39.4 \%$ \\
\hline Electroencephalography & Normal & Normal \\
\hline
\end{tabular}

In conclusion, polysomnography showed a prominent pattern of central apnoeas and periodic breathing with mild desaturations. Central apnoea is defined as the absence of both airflow and respiratory effort. Central apnoeas of $<20 \mathrm{~s}$ are a normal phenomenon in children. Furthermore, these central events are almost never accompanied by oxygen desaturation and a desaturation below 89\% following a central apnoea occurrence is considered to be abnormal [1]. The clustering of central apnoeas, periodic breathing, is a normal phenomenon in infants, especially in premature infants but normally disappears during the first months of life.

\section{Question 2: What is your differential diagnosis?}

a) Late-onset congenital central hypoventilation syndrome

b) Idiopathic central sleep apnea

c) Central nervous system pathology

d) Inborn errors of metabolism

e) Neuromuscular disease

f) Restrictive lung disorders

g) Cardiac dysfunction

h) Drugs

i) Hormonal disorders 


\section{Answer 2:}

Since we had a report of a normal brain MRI performed in the last 6 months, central nervous pathology was less probable. Neurological examination was also normal, therefore, neuromuscular disease was unlikely. Therefore, the correct possible answers are $a_{1}$ $b, d, f, g, h$ and i. Idiopathic central sleep apnoea requires the absence of all other disorders. There were no other symptoms or clinical signs to support answer $d, f, g, h$ and $i$. Other causes of central apnoea in children include obesity-hypoventilation syndrome and syndromal causes, including Joubert syndrome, which were unlikely in our patient because of a normal body mass index, the absence of dysmorphic features and normal developmental milestones.

The differential diagnosis of pathological central sleep apnoea in children is broad but is also characterised by the absence of diagnostic guidelines. Although we had a report of a normal brain MRI, we suspected a central nervous system pathology, also in view of the previous headache problems. However, we performed several investigations to rule out other causes because of the marked abnormalities on polysomnography and because the MRI images had to be transferred from another hospital

Chest radiograph and lung function testing were normal. Cardiac workup with ultrasound and electrocardiogram was normal. Laboratory evaluation showed a normal blood gas, complete blood cell count, kidney and liver function,

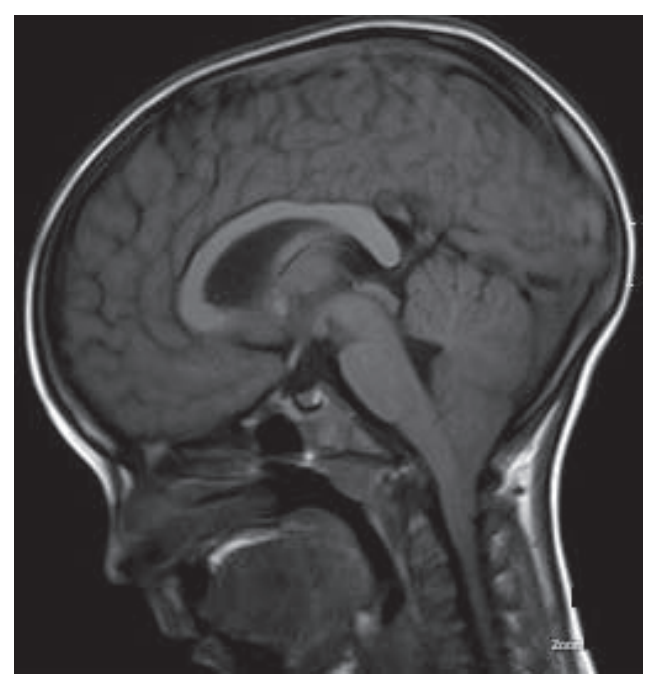

Figure 2

MRI scan.

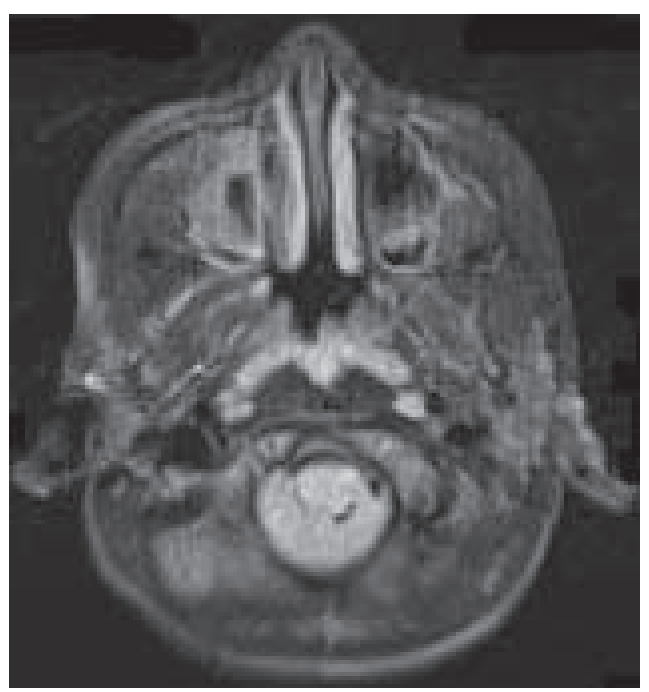

Figure 3

MRI scan.

creatinine kinase, lactate and ammonia levels. Analysis of cerebrospinal fluid showed no abnormalities with a normal cell count. Metabolic investigation of urine, cerebrospinal fluid and plasma was completely normal. PHOX2B gene testing for late-onset congenital central hypoventilation was negative. Toxicological screening was negative. In view of the severe elevated percentage of periodic breathing during sleep and because all results came back normal, we decided to repeat the MRI scan of brain and spine (figs 2 and 3).

\section{Question 3: What does the MRI brain scans show?}
a) Brain stem tumour
b) Normal
c) Chiari malformation 


\section{Answer 3:}

c) The MRI shows an Arnold Chiari malformation typel, which is defined as a downward displacement of the cerebellar tonsils and medulla through the foramen magnums

The brain MRI showed that the periodic breathing in our patient was caused by a central nervous system abnormality, namely an Arnold Chiari malformation. The scan also showed a normal structure of the cervical spine. The patient was referred to neurosurgery for decompression of the fossa posterior. Meanwhile, our patient was started on bilevel non-invasive ventilation through a nasal mask. After a titration night, the patient was on the following settings: IPAP 9, EPAP 5 and back-up rate of 14 . BiPAP ventilation normalised the nocturnal breathing pattern and his sleep architecture. On bilevel ventilation, our patient also felt more refreshed on awakening and had more energy during the daytime.

\section{Discussion}

Arnold Chiari malformation is a known cause of central apnoea. Its prevalence is estimated to be $0.1-0.5 \%$. It can be asymptomatic, but sometimes headache is involved. The type-1 malformation is characterised by caudal herniation of the cerebellar tonsils through the foramen magnum. It rarely presents before adolescence [2]. Furthermore, a presentation with isolated sleep apnoea is relatively infrequent [3], although several cases of sleep apnoea syndrome as the sole neurologic manifestation of Chiari malformation type- have been reported. Thus, the presence of pathological central apnoeas in otherwise well children merits a thorough investigation of brain and cervical MRI as is illustrated in our case report [3]. Type-2 is characterised by displacement of the cerebellar vermis, caudal brainstem and fourth ventricle through the foramen magnum.

Chiari malformation results in an abnormal breathing pattern because the brainstem respiratory centres are affected resulting in an abnormal central control of breathing. This can be due to abnormal brainstem nuclei or mechanical compression of the brainstem [4]. Sleep apnoea is present in $67 \%$ of patients with Chiari malformation [5]. These patients also have an increased risk of sudden death, presumably due to prolonged apnoea [6]. Children with Chiari malformation frequently have sleep-related respiratory abnormalities even in the absence of symptoms. When the diagnosis is made, posterior fossa decompression is warranted [7].

\section{References}

1. Verhulst SL, Schrauwen N, Haentjens D, et al. Reference values for sleep-related respiratory variables in asymptomatic European children and adolescents. Pediatr Pulmonol 2007; 42: 159-167.

2. Murray C, Seton C, Prelog K, et al. Arnold Chiari type 1 malformation presenting with sleep disordered breathing in well children. Arch Dis Child 2006; 91: 342-343.

3. Spence J, Pasterkamp H, McDonald PJ. Isolated central sleep apnea in type I Chiari malformation: improvement after surgery. Pediatr Pulmonol 2010; 45: 1141-1144.

4. Carroll JL, Marcus CL, Loughlin GM. Sleep and breathing in children: A developmental approach, New York: Marcel Dekker, 2000; 532-535.

5. Dauvilliers Y, Stal V, Abril B, et al. Chiari malformation and sleep related breathing disorders. J Neurol Neurosurg Psychiatry 2007; 78: 1344-1348.

6. Hays RM, Jordan RA, McLaughlin JF, et al. Central ventilatory dysfunction in myelodysplasia: an independent determinant of survival. Dev Med Child Neurol 1989; 31: 366-370.

7. Hershberger ML, Chidekel A. Arnold-Chiari malformation type I and sleep-disordered breathing: an uncommon manifestation of an important pediatric problem. J Pediatr Health Care 2003; 17: 190-197. 